\title{
Incidence of hypertension among persons living with HIV in China: a multicenter cohort study
}

Hongwei Fan ${ }^{1 \dagger}$, Fuping Guo ${ }^{1 \dagger}$, Evelyn Hsieh ${ }^{1,2}$, Wei-Ti Chen ${ }^{3}$, Wei Lv ${ }^{1}$, Yang Han ${ }^{1}$, Jing Xie ${ }^{1}$, Yanling Li ${ }^{1}$, Xiaojing Song ${ }^{1}$ and Taisheng $\mathrm{Li}^{1,4^{*}}$

\begin{abstract}
Background: Life expectancy among persons living with HIV (PLWH) has improved with increasing access to antiretroviral therapy (ART), however incidence of chronic comorbidities has simultaneously increased. No data are available regarding the incidence of hypertension among Chinese PLWH.

Methods: We analyzed data collected from patients enrolled in two prospective longitudinal multicenter studies of PLWH initiating ART in China. Incidence rate of hypertension per 100 person-years (PYs) among PLWH was calculated, and Cox proportional hazards models was used to evaluate the association between incident hypertension and traditional and HIVassociated risk factors.

Results: Of 1078 patients included in this analysis, 984 ART-naïve patients were hypertension-free at baseline, and contributed 2337.7 PYs of follow up, with a median follow-up period of 1.8 years (range: 1.2-3.2) after initiation of ART. Incidence of hypertension was 7.6 [95\% confidence interval (CI): 6.5-8.7] per 100 PYs. In the Cox regression analysis, incidence of hypertension was positively associated with body mass index [adjusted hazard ratio (aHR) $1.07(1.01,1.13), p=0.02$ ] and recent viral load (aHR 1.28, 95\% Cl:1.08-1.51, $p<0.01$ ), and negatively associated with recent CD4+/CD8+ ratio (aHR 0.14, 95\% Cl:0.06-0.31, $p<0.001$ ), zidovudine exposure (aHR 0.15, 95\% Cl: $0.10-0.24, p<0.001$ ) and tenofovir disoproxil fumarate exposure (aHR 0.13, 95\% Cl: $0.08-0.21, p<0.001)$.

Conclusions: The incidence of hypertension was relatively high among Chinese PLWH initiating ART. Recent low CD4+/CD8+ ratio and detectable HIV viremia were associated with incident hypertension, whereas receipt of ART was associated with reduced risk. Hypertension may be mitigated, in part, by excellent HIV care, including viral suppression with ART.
\end{abstract}

Trial registration: ClinicalTrials.gov Identifier: NCT00872417 registered on 31 March, 2009, and NCT01844297 registered on 1 May, 2013.

Keywords: HIV, Hypertension, Incidence

\footnotetext{
* Correspondence: litsh@263.net

${ }^{\dagger}$ Hongwei Fan and Fuping Guo contributed equally to this work.

'Department of Infectious Diseases, Peking Union Medical College Hospital,

Chinese Academy of Medical Sciences, Beijing, China

${ }^{4}$ Center for AIDS Research, Chinese Academy of Medical Sciences and Peking

Union Medical College, Beijing, China

Full list of author information is available at the end of the article
}

(C) The Author(s). 2020 Open Access This article is licensed under a Creative Commons Attribution 4.0 International License, which permits use, sharing, adaptation, distribution and reproduction in any medium or format, as long as you give appropriate credit to the original author(s) and the source, provide a link to the Creative Commons licence, and indicate if changes were made. The images or other third party material in this article are included in the article's Creative Commons licence, unless indicated otherwise in a credit line to the material. If material is not included in the article's Creative Commons licence and your intended use is not permitted by statutory regulation or exceeds the permitted use, you will need to obtain permission directly from the copyright holder. To view a copy of this licence, visit http://creativecommons.org/licenses/by/4.0/. The Creative Commons Public Domain Dedication waiver (http://creativecommons.org/publicdomain/zero/1.0/) applies to the data made available in this article, unless otherwise stated in a credit line to the data. 


\section{Background}

Due to the increased life expectancy of persons living with HIV (PLWH) after successful antiretroviral therapy (ART), management of aging-related non-communicable diseases (including cardiovascular disease, diabetes, chronic kidney disease, osteoporosis, and non-AIDS malignancies) has become a routine part of HIV care [1]. Hypertension is a key risk factor for cardiovascular diseases and is prevalent among PLWH [2]. A recent metaanalysis, including 49 studies with 63,554 participants from America, Europe, Africa and Asia from 1996 to 2014, reported an estimated hypertension prevalence of $12.7 \%$ for ART-naïve and $34.7 \%$ for ART-experienced participants [3]. Epidemiological studies from the United States and several European countries have demonstrated incidence of hypertension among outpatient primary care and HIV clinics for PLWH ranging from 2.6 to 7.2 per 100 person-years (PYs) [4-9]. Studies from East Africa have reported incidence rates of 11.2-12.0 per 100 PYs [10, 11].

Limited studies have focused on hypertension prevalence among PLWH in Asia [12-14], and these have all been cross-sectional in design. We previously reported the prevalence of hypertension was $8.4 \%$ among Chinese ART-naïve PLWH [12], which is lower than that reported in the USA and Europe [3]. However, the incidence of hypertension among PLWH in Asia, particularly after initiation of ART, remains unclear.

The risk factors contributing to hypertension among PLWH are multifactorial, controversial, and include traditional contributors (e.g. older age, male sex, obesity, family history, smoking, comorbidities) $[5-8,10,11]$, and, in some studies, HIV-related factors including immunodeficiency [11], longer duration of HIV infection or advanced HIV clinical stage [4, 6], and ART [15]. However, other studies have reported no association between HIV-related factors and hypertension risk among ART-experienced PLWH $[8,10]$.

In order to provide important data regarding hypertension incidence among Chinese PLWH after initiation of ART, and to examine the association of traditional and HIV-associated risk factors with incident hypertension in this population, we designed the present analysis, leveraging data collected as part of two large prospective multicenter studies among Chinese PLWH.

\section{Methods}

\section{Study Design \& Population}

We performed a secondary analysis of data collected as part of two large prospective multicenter studies of adult patients with HIV in China carried out by the same collaborative research network. These studies recruited patients from regions of high HIV prevalence across China, and were established to systematically evaluate the efficacy, toxicities and co-morbidities associated with the first-line government-sponsored free ART regimens available at the time each study was initiated.

In brief, the first study, [China AIDS Clinical Trial (CACT) 1810; clinicaltrials.gov ID: NCT00872417], initiated in 2008, was carried out in 8 cities across China (Beijing, Shanghai, Zhengzhou, Fuzhou, Guangzhou, Shenzhen, Xi'an and Yunnan), and recruited a total of 543 treatment-naïve adult patients with HIV from November 8, 2008 to August 6, 2010 [16]. This was an open-label trial comparing the efficacy and safety of three different ART treatment regimens over 96 weeks using government-sponsored first-line agents in 2008: Stavudine (d4T, $30 \mathrm{mg}$ twice daily, Desano, Shanghai, China) or Zidovudine (AZT, $300 \mathrm{mg}$ twice daily, Northeast General Pharmaceutical Factory, China) plus lamivudine (3TC, $300 \mathrm{mg}$ once daily, GlaxoSmithKline, UK) and nevirapine (NVP, $200 \mathrm{mg}$ once daily for the first 2 weeks and then $200 \mathrm{mg}$ twice daily, Desano) or efavirenz (EFV, $600 \mathrm{mg}$ once daily, MSD, Australia). Subsequent funding enabled an extension of this study beyond the initial 96-week follow-up period through the time of the present analysis.

The second study, (CACT1215 clinicaltrials.gov ID: NCT01844297), initiated in 2012, was carried out in 9 cities across China (Beijing, Shanghai, Guangzhou, Chengdu, Changsha, Nanning, Liuzhou, Zhengzhou, Shenyang) and recruited a total of 583 treatment-naïve adult patients with HIV from July 17, 2012 to July 3, 2014. This study was a cohort study examining the efficacy and safety at 96 weeks of the current first-line ART treatment regimen in China [tenofovir disoproxil fumarate (TDF, $300 \mathrm{mg}$ once daily, Gilead Sciences, Inc., USA) plus $3 \mathrm{TC}$ and EFV]. Subsequent funding also allowed an extension of this study beyond the initial 96-week follow up period through the time of the present analysis.

Both studies collected sociodemographic data, clinical data related to HIV and HIV-related risk factors, and serologic biospecimens. Eligibility criteria at baseline included age 18-65 years, confirmed HIV-infection status via Western blot, CD4+ cell count $<350$ cells $/ \mathrm{mm}^{3}$ (for CACT1810) or $<500$ cells $/ \mathrm{mm}^{3}$ (for CACT1215), and lack of prior exposure to ART. After enrollment, participants initiated treatment with ART, and returned for follow-up evaluations at weeks $2,4,8,12,24$, and once every 12 weeks thereafter for clinical and laboratory evaluations. Trained study staff at each study site performed detailed clinical evaluations and recorded data in an electronic study database.

Peking Union Medical College Hospital (PUMCH) in Beijing, China served as the primary study center for both studies, and training and monitoring of study sites was overseen by the same contract research organization. Both protocols were approved by the 
PUMCH institutional review board prior to initiation of study activities. All study participants provided written informed consent at the time of enrollment, and all procedures were performed in compliance with the ethical standards of The Declaration of Helsinki.

For the present analysis, we included patients enrolled in both parent studies in order to encompass ART treatment regimens utilized in China over the past decade. Patients who did not complete at least 2 visits (including the baseline visit) were excluded from our analysis (database cutoff date June 2015).

\section{Definitions}

Hypertension was defined based upon the Joint National Committee on Prevention, Detection, Evaluation and Treatment of High Blood Pressure Recommendations [17], namely a systolic blood pressure $\geq 140 \mathrm{mmHg}$ or diastolic blood pressure $\geq 90 \mathrm{mmHg}$ at two different time points, or receiving a prescription for antihypertensive medication. Trained study personnel at each site performed standardized blood pressure measurements using a sphygmomanometer at each study encounter. For each participant, blood pressure was measured using a manual sphygmomanometer with appropriate cuff size based on arm circumference after more than $5 \mathrm{~min}$ of seated rest and without smoking, exercise or feeding. One measurement was taken positioned at the level of the heart, and recorded as the blood pressure value.

We classified body mass index (BMI) using the following categories: underweight $<18.5$, normal 18.5-24.9, overweight $25-29.9$, and obese $\geq 30.0 \mathrm{~kg} / \mathrm{m}^{2}$ [18]; and dyslipidemia using the following categories: total cholesterol $>5.2 \mathrm{mmol} / \mathrm{l}$, high-density lipoprotein cholesterol (HDL-c) $<1.0 \mathrm{mmol} / \mathrm{l}$, low-density lipoprotein cholesterol $>4.1 \mathrm{mmol} / \mathrm{l}$, or triglycerides $>1.7 \mathrm{mmol} / \mathrm{l}$ [19]. Participants were classified as diabetic if they had a prior diagnosis of diabetes, a fasting plasma glucose $\geq 7.0$ $\mathrm{mmol} / \mathrm{l}$ or were being treated with insulin or oral hypoglycemic agents [12]. Renal function was assessed by the estimated glomerular filtration rate (eGFR) using the formula from the Chronic Kidney Disease Epidemiology Collaboration [20], and categorized following the chronic kidney disease criteria [21].

Data from each study encounter regarding CD4+ and CD8+ T-cell levels and CD4+/CD8+ ratios were collected and we defined recent $\mathrm{VL}$, recent $\mathrm{CD} 4+$ cell counts and recent $\mathrm{CD} 4+/ \mathrm{CD} 8+$ ratio as the laboratory values obtained at the visit when hypertension was diagnosed, or, for those who did not develop hypertension, the last study encounter. HIV-1 viral load (VL) measurements, performed using the Cobas AmpliPrep/Cobas TaqMan real-time RT-PCR Assay (Roche, CA, USA), were also collected. Undetectable VL results were given the value of the detection limit (20 copies $/ \mathrm{ml})$. Complete and incomplete viral suppression were defined as HIV $\mathrm{VL}<50$ and $<400$ copies $/ \mathrm{mL}$, respectively.

\section{Statistical analysis}

We tabulated and reported descriptive data using means \pm standard deviations and frequencies. The Student's $t$-test for parametric continuous variables, Mann-Whitney $U$ test for non-parametric continuous variables, and the Chi-squared test for categorical variables were used to compare the clinical characteristics between patients with and without hypertension. Incidence rates of hypertension per 100 PYs during the observation period were calculated. Stepwise Cox regression analysis was used to estimate hazard ratios (HR) with 95\% confidence intervals (CI). Patients were censored at time of incident of hypertension, loss to follow-up, death, withdrawal from study during follow-up or end of study. The observation period began at the enrollment visit and ended at the date of hypertension diagnosis or the last clinical encounter for those who did not develop hypertension. Participants with hypertension at baseline were excluded from the longitudinal analyses.

Covariates analyzed included age, sex, BMI, Han ethnicity, current smoking, current alcohol use, eGFR, diabetes, dyslipidemia, hepatitis B virus (HBV) surface antigen (HBsAg), hepatitis $\mathrm{C}$ virus (HCV) antibody ( $\mathrm{HCV} \mathrm{Ab),} \mathrm{route} \mathrm{of} \mathrm{HIV} \mathrm{transmission,} \mathrm{years} \mathrm{since} \mathrm{HIV}$ diagnosis, baseline $\mathrm{CD} 4+$ cell count, baseline VL, stavudine, zidovudine, and TDF exposure, recent CD4+ cell count, recent $\mathrm{CD} 4+/ \mathrm{CD} 8+$ ratio, and recent VL. All statistical analyses were performed using SPSS 19.0 statistical software package (IBM Corporation, Armonk, New York, USA) and Prism version 6 (GraphPad Software, Inc., La Jolla, CA). For all tests, $p<0.05$ was considered statistically significant.

\section{Results \\ Population characteristics}

Out of 1126 total patients enrolled in the parent studies, we excluded forty-eight patients who withdrew after the initial evaluation, yielding 1078 patients eligible for analysis (Fig. 1). Of the included participants (mean age $35.7 \pm 10.1$ years, $75.0 \%$ men), Han Chinese comprised $84.9 \%$ of individuals, current smokers represented 23.9, 12.2 and $7.3 \%$ were co-infected with HBV and HCV respectively, and men who have sex with men (MSM) represented $39.4 \%$ of the population. The baseline CD4+ cell count was $234 \pm 124$ cells $/ \mathrm{mm}^{3}$, and the baseline VL was $4.7 \pm 0.7 \log 10$ copies $/ \mathrm{ml}$ (Table 1$)$.

At baseline (Table 1), 94 participants had a diagnosis of hypertension [8.7, 95\% CI 7.0-10.4\%]. When compared with the 984 participants without hypertension, those with hypertension at baseline were older (41.0 vs. 


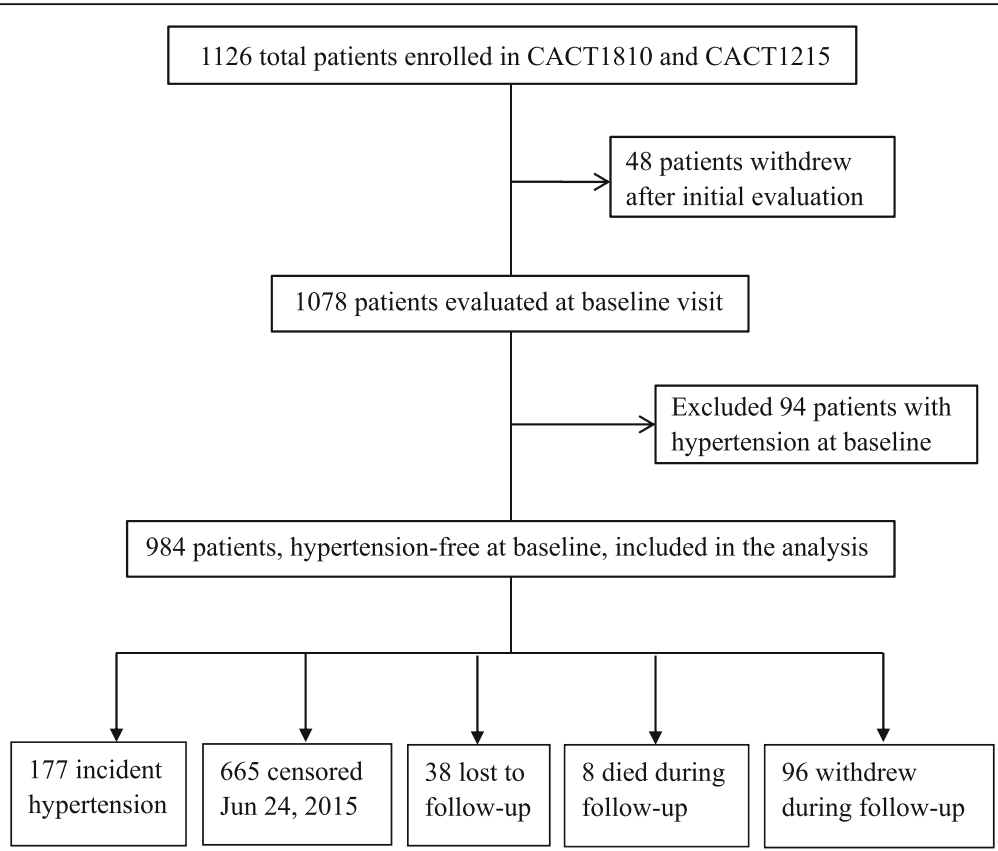

Fig. 1 Patient flow chart. Abbreviation: CACT: China AIDS Clinical Trial

35.2 years), had higher BMI levels (22.8 vs. $\left.21.5 \mathrm{~kg} / \mathrm{m}^{2}\right)$, lower eGFR (108 vs. $114 \mathrm{ml} / \mathrm{min}$ ), lower prevalence of HDL-c $<1.0 \mathrm{mmol} / \mathrm{l}(21.7 \%$ vs. $31.8 \%)$, and higher prevalence of HBsAg+ $(19.1 \%$ vs. $11.6 \%)$ (All $p<0.05)$. In addition, patients with hypertension at baseline were much less likely to have acquired HIV via homosexual transmission as compared with other routes. Finally, no differences between the two groups were found with respect to years since HIV diagnosis, baseline CD4+ cell count and VL.

The 984 participants included in the longitudinal study (Table 1 and Table 2) had a median follow up duration of 1.8 years (interquartile range, 1.2, 3.2 years; range: 0.01-6.3 years), and the following baseline characteristics: mean age $35.2 \pm 9.9$ years, $75.2 \%$ male, $84.7 \%$ of Han ethnicity, $40.7 \%$ MSM and $24.2 \%$ current smokers. Baseline CD4+ cell count was $233 \pm 125$ cells $/ \mathrm{mm}^{3}$, and HIV VL was $4.7 \pm 0.7 \log 10$ copies $/ \mathrm{ml}$. The prevalence of participants co-infected with hepatitis B and hepatitis C were 11.6 and $7.3 \%$ respectively. At the time of hypertension diagnosis or the last encounter for those who did not develop hypertension, median CD4+ cell count and VL were $431 \pm 203$ cells $/ \mathrm{mm}^{3}$ and $1.6 \pm 0.7 \log 10$ copies/ml respectively. Complete and incomplete viral suppression were achieved by 78.2 and $87.6 \%$ of participants, respectively.

At the database cutoff date (Fig. 1), 142 (14.4\%) participants were no longer being followed for the following reasons: 8 participants died (one car accident, one liver cirrhosis, one lactic acidosis, two with opportunistic infection, two with cerebral hemorrhage, and one with unknown cause of death), 96 participants had withdrawn from the study [16 participants with virologic failure, 9 participants experienced severe adverse events (one opportunistic infection, one toxoplasma encephalopathy, one hepatotoxicity, two with rash, four with bone marrow suppression), 71 participants voluntarily withdrew from the study], and 38 participants were lost to follow-up.

\section{Incidence of hypertension}

The 984 study participants included in the longitudinal analysis contributed a total of 2337.7 PYs of follow-up. One hundred seventy-seven participants developed hypertension during the follow-up period, yielding an incidence of 7.6 (95\% CI: 6.5-8.7) per 100 PYs. When stratified by cohort, a total of 476 patients from CACT1810 contributed 1549.95 PYs of follow-up (median follow-up time of 3.9 years) and 123 patients developed hypertension during this time. A total of 508 patients from CACT1215 contributed 787.72 PYs (median follow-up time of 1.8 years) and 54 patients developed hypertension. The incidence of hypertension was not significantly different between the participants in the two groups [7.9 (95\% CI: 6.6-9.2) v. 6.9 (95\% CI: $5.1-8.7)$ per 100 PYs, respectively $(p=0.35)]$.

\section{Risk factors for incident hypertension}

Table 3 shows the results of our univariate and multivariable regression analyses. In the multivariable Cox regression model, for every $1 \mathrm{~kg} / \mathrm{m}^{2}$ increase in BMI, we observed a $7 \%$ increase in the incidence of hypertension 
Table 1 Demographic and Clinical Characteristics, Stratified by Baseline Hypertension Status ( $N=1078)$

\begin{tabular}{|c|c|c|c|c|}
\hline Characteristic & $\begin{array}{l}\text { Total } \\
(n=1078)\end{array}$ & $\begin{array}{l}\text { No HTN } \\
(n=984)\end{array}$ & $\begin{array}{l}\text { Baseline HTN } \\
(n=94)\end{array}$ & $P$ value \\
\hline Male & $808(75.0)$ & $740(75.2)$ & $68(72.3)$ & 0.541 \\
\hline Age (years) & $35.7 \pm 10.1$ & $35.2 \pm 9.9$ & $41.0 \pm 10.8$ & $<0.001$ \\
\hline $\operatorname{BMI}\left(\mathrm{kg} / \mathrm{m}^{2}\right)$ & $21.6 \pm 2.9$ & $21.5 \pm 2.9$ & $22.8 \pm 3.0$ & $<0.001$ \\
\hline Overweight & $113(10.5)$ & $93(9.5)$ & $20(21.3)$ & $<0.001$ \\
\hline Obese & $11(1.0)$ & $10(1.0)$ & $1(1.1)$ & NA \\
\hline Han ethnicity & $915(84.9)$ & $833(84.7)$ & $82(87.2)$ & 0.505 \\
\hline Current smoking & $258(23.9)$ & $238(24.2)$ & $20(21.3)$ & 0.527 \\
\hline Current alcohol use & $288(26.7)$ & $264(26.8)$ & $24(25.5)$ & 0.786 \\
\hline eGFR $(\mathrm{ml} / \mathrm{min})$ & $113 \pm 17$ & $114 \pm 17$ & $108 \pm 17$ & 0.002 \\
\hline $\mathrm{eGFR}<90(\mathrm{~m} / / \mathrm{min})$ & $98(9.3)$ & $83(8.6)$ & $15(16.3)$ & 0.015 \\
\hline Diabetes & $51(4.8)$ & $49(5.1)$ & $2(2.2)$ & 0.21 \\
\hline $\mathrm{TC}>5.2(\mathrm{mmo} / \mathrm{l})$ & $111(10.5)$ & $102(10.6)$ & $9(9.8)$ & 0.81 \\
\hline $\mathrm{TG}>1.7(\mathrm{mmo} / \mathrm{l})$ & 313 (29.6) & $283(29.4)$ & $30(32.6)$ & 0.51 \\
\hline $\mathrm{HDL}-\mathrm{c}<1.0(\mathrm{mmol} / \mathrm{l})$ & $323(30.9)$ & 303 (31.8) & $20(21.7)$ & 0.046 \\
\hline LDL-c $>4.1(\mathrm{mmol} / \mathrm{l})$ & $18(1.7)$ & $14(1.5)$ & $4(4.3)$ & NA \\
\hline Dyslipidemia & $541(52.2)$ & $497(52.6)$ & $44(47.8)$ & 0.38 \\
\hline $\mathrm{HBs} \mathrm{Ag}+$ & $127(12.2)$ & 110 (11.6) & $17(19.1)$ & 0.04 \\
\hline $\mathrm{HCV}-\mathrm{Ab}+$ & $75(7.3)$ & $69(7.3)$ & $6(6.6)$ & 0.79 \\
\hline Route of HIV transmission & & & & 0.01 \\
\hline Heterosexual & $497(46.1)$ & $451(45.8)$ & $46(48.9)$ & \\
\hline Homosexual & $425(39.4)$ & $400(40.7)$ & 25 (26.6) & \\
\hline Blood transfusion & $53(4.9)$ & $45(4.6)$ & $8(8.5)$ & \\
\hline Others & $103(9.6)$ & $88(8.9)$ & $15(16.0)$ & \\
\hline Years since HIV diagnosis & $1.0 \pm 1.7$ & $1.0 \pm 1.7$ & $1.1 \pm 1.6$ & 0.72 \\
\hline CD4+ cell count $\left(\right.$ cells $\left./ \mathrm{mm}^{3}\right)$ & $234 \pm 124$ & $233 \pm 125$ & $251 \pm 114$ & 0.18 \\
\hline$<200$ & $413(38.5)$ & $384(39.1)$ & $29(31.2)$ & 0.19 \\
\hline $200-350$ & $461(42.9)$ & $413(42.1)$ & $48(51.6)$ & \\
\hline $350-499$ & $200(18.6)$ & $184(18.8)$ & $16(17.2)$ & \\
\hline Viral load (log10 copies/ml) & $4.7 \pm 0.7$ & $4.7 \pm 0.7$ & $4.6 \pm 0.7$ & 0.15 \\
\hline$<5$ & $571(67.3)$ & $517(67.3)$ & $54(66.7)$ & 0.91 \\
\hline$\geq 5$ & $278(32.7)$ & $251(33.2)$ & 27 (33.3) & \\
\hline
\end{tabular}

All characteristics measured at baseline

Values are shown as $\mathrm{n}(\%)$ or mean \pm standard deviation

Abbreviations: HTN hypertension; $B M I$ body mass index; $N A$, not available; eGFR, estimated glomerular filtration rate; $T C$ total cholesterol; $T G$, triglycerides; $H D L-C$, high-density lipoprotein cholesterol; $L D L-C$, low-density lipoprotein cholesterol; $H B s A g+$, hepatitis B surface antigen; $H C V$ - $A b$, hepatitis $C$ antibody; $H I V$, human immunodeficiency virus

[adjusted HR (aHR) 1.07, 95\% CI:1.01-1.13, $p=0.02$ ]. Zidovudine exposure (aHR 0.15, 95\% CI: $0.10-0.24, p<$ 0.001 ) and TDF exposure (aHR 0.13, 95\% CI: $0.08-0.21$, $\mathrm{p}<0.001)$ correlated with a lower risk of hypertension. At the time of hypertension diagnosis or the last encounter (for those who did not develop hypertension), a lower recent $\mathrm{CD} 4+/ \mathrm{CD} 8+$ ratio (aHR 0.14, 95\% CI: $0.06-0.31, \mathrm{p}<0.001)$ and a higher recent VL (aHR 1.28, 95\% CI: $1.08-1.51, p<0.01)$ were also associated with incident hypertension (Fig. 2).

\section{Discussion}

This study is the first to report incidence of hypertension among Chinese PLWH and to evaluate risk factors associated with incident hypertension in this population. We found that hypertension incidence was 7.6 (95\% CI: 6.5-8.7) per 100 PYs, and higher incidence was significantly associated with specific traditional (high BMI), and HIV-related risk factors (higher recent VL, lower recent $\mathrm{CD} 4+/ \mathrm{CD} 8+$ ratio, lack of exposure of TDF or zidovudine). 
Table 2 Demographic and Clinical Characteristics, Stratified by Incident Hypertension Status ( $N=984)$

\begin{tabular}{|c|c|c|c|}
\hline Characteristic & No $H T N(n=807)$ & HTN $(n=177)$ & $P$ value \\
\hline Male & $603(74.7)$ & $137(77.4)$ & 0.46 \\
\hline Age (years) & $34.4 \pm 9.4$ & $38.8 \pm 11.2$ & $<0.001$ \\
\hline BMI $\left(\mathrm{kg} / \mathrm{m}^{2}\right)$ & $21.3 \pm 2.8$ & $22.1 \pm 3.1$ & $<0.01$ \\
\hline Overweight & $72(8.9)$ & $21(11.9)$ & 0.23 \\
\hline Obese & $6(0.7)$ & $4(2.3)$ & NA \\
\hline Han ethnicity & $676(83.8)$ & $157(88.7)$ & 0.10 \\
\hline Smoking & $200(24.8)$ & $38(21.5)$ & 0.35 \\
\hline Current alcohol use & $224(27.8)$ & $40(22.6)$ & 0.16 \\
\hline eGFR $(\mathrm{ml} / \mathrm{min})$ & $114 \pm 16$ & $110 \pm 19$ & $<0.01$ \\
\hline eGFR < $90(\mathrm{ml} / \mathrm{min})$ & $62(7.7)$ & $21(11.9)$ & 0.07 \\
\hline Diabetes & $40(5.0)$ & $9(5.1)$ & 0.95 \\
\hline $\mathrm{TC}>5.2(\mathrm{mmo} / \mathrm{l})$ & $81(10.0)$ & $21(11.9)$ & 0.48 \\
\hline $\mathrm{TG}>1.7(\mathrm{mmo} / \mathrm{l})$ & $229(28.4)$ & $54(30.5)$ & 0.59 \\
\hline $\mathrm{HDL}-\mathrm{c}<1.0(\mathrm{mmol} / \mathrm{l})$ & $240(29.7)$ & $63(35.6)$ & 0.17 \\
\hline $\mathrm{LDL}-\mathrm{c}>4.1(\mathrm{mmol} / \mathrm{l})$ & $11(1.4)$ & $3(1.7)$ & NA \\
\hline Dyslipidemia & $397(49.2)$ & $100(56.5)$ & 0.15 \\
\hline $\mathrm{HBs} \mathrm{Ag}+$ & $88(10.9)$ & $22(12.4)$ & 0.51 \\
\hline $\mathrm{HCV} \mathrm{Ab}+$ & $50(6.2)$ & $19(10.7)$ & 0.03 \\
\hline Route of HIV transmission & & & $<0.001$ \\
\hline Heterosexual & $339(42.0)$ & $61(34.5)$ & \\
\hline Homosexual & $370(45.8)$ & $81(45.8)$ & \\
\hline Blood transfusion & $23(2.9)$ & $22(12.4)$ & \\
\hline Others & $75(9.3)$ & $13(7.3)$ & \\
\hline Years since HIV diagnosis & $1.0 \pm 1.7$ & $1.1 \pm 1.8$ & 0.36 \\
\hline Baseline CD4+ cell count (cells/mm³) & $238 \pm 125$ & $206 \pm 124$ & $<0.01$ \\
\hline $\begin{array}{l}\text { Baseline HIV Viral load } \\
\text { (log10 copies } / \mathrm{ml})\end{array}$ & $4.7 \pm 0.7$ & $4.7 \pm 0.7$ & 0.52 \\
\hline Stavudine exposure & $248(30.7)$ & $97(54.8)$ & $<0.001$ \\
\hline $\begin{array}{l}\text { Years of stavudine exposure } \\
\text { Zidovudine exposure }\end{array}$ & $\begin{array}{l}1.2 \pm 1.1 \\
316(39.2)\end{array}$ & $\begin{array}{l}0.6 \pm 0.7 \\
64(36.2)\end{array}$ & $<0.0010 .46$ \\
\hline $\begin{array}{l}\text { Years of zidovudine exposure } \\
\text { TDF exposure }\end{array}$ & $\begin{array}{l}3.3 \pm 1.9 \\
470(58.2)\end{array}$ & $\begin{array}{l}1.2 \pm 1.1 \\
54(30.5)\end{array}$ & $\begin{array}{l}<0.001 \\
<0.001\end{array}$ \\
\hline $\begin{array}{l}\text { Years of TDF exposure } \\
\text { Recent CD4+ cell count (cells/mm })\end{array}$ & $\begin{array}{l}1.8 \pm 0.7 \\
453 \pm 204\end{array}$ & $\begin{array}{l}0.4 \pm 0.5 \\
330 \pm 162\end{array}$ & $\begin{array}{l}<0.001 \\
<0.001\end{array}$ \\
\hline Recent CD4+/CD8+ ratio & $0.7 \pm 0.5$ & $0.5 \pm 0.3$ & $<0.001$ \\
\hline Recent VL (log10 copies/ml) & $1.5 \pm 0.7$ & $2.0 \pm 0.7$ & $<0.001$ \\
\hline
\end{tabular}

Values are shown as $\mathrm{n}(\%)$ or mean \pm standard deviation

Abbreviations: HTN hypertension; BMI body mass index; NA not available; $e G F R$, estimated glomerular filtration rate; TC total cholesterol; TG triglycerides; $H D L-C$ high-density lipoprotein cholesterol; $L D L-C$, low-density lipoprotein cholesterol; $H B s A g+$, hepatitis B surface antigen; $H C V-A b$ hepatitis $C$ antibody; $H I V$ human immunodeficiency virus; VL HIV-1 viral load; TDF tenofovir disoproxil fumarate

* Unless stated otherwise, characteristics reported represent baseline characteristics

While hypertension is commonly seen among PLWH, data conflict regarding whether hypertension is more prevalent among ART-naive PLWH compared with HIV-negative controls, as there is significant heterogeneity across different study designs [22]. The prevalence of hypertension observed among ART-naive PLWH in the present study was lower than that reported in the
Chinese general population (26.9\%), among a nationally representative sample of over 90,000 Chinese adults from 2007 to 2008 [23]. This might be attributable to younger age, lower BMI and prevalence of smoking among Chinese ART-naïve PLWH in the present study compared with the general population cohort, or to differences in other risk factors between the time periods 
Table 3 Cox Regression Analysis of Association between Clinical Risk Factors and Incidence of Hypertension

\begin{tabular}{|c|c|c|c|c|}
\hline Covariate & $\begin{array}{l}\text { Univariate model } \\
\text { HR }(95 \% \mathrm{CI})\end{array}$ & $P$ value & $\begin{array}{l}\text { Multivariable model } \\
\text { HR }(95 \% \mathrm{Cl})\end{array}$ & $P$ value \\
\hline Male & $1.14(0.80,1.62)$ & 0.47 & & \\
\hline Age (per year increase) & $1.04(1.03,1.05)$ & $<0.001$ & $1.02(1.00,1.03)$ & 0.06 \\
\hline BMI (per kg/m² increase) & $1.09(1.04,1.14)$ & $<0.001$ & $1.07(1.01,1.13)$ & 0.02 \\
\hline Han ethnicity & $1.27(0.80,2.02)$ & 0.32 & & \\
\hline Current smoking & $0.84(0.59,1.21)$ & 0.35 & & \\
\hline Current alcohol use & $0.83(0.58,1.18)$ & 0.30 & & \\
\hline eGFR (per ml/min increase) & $0.99(0.98,1.00)$ & $<0.01$ & NA & NA \\
\hline Diabetes & $1.19(0.61,2.32)$ & 0.62 & & \\
\hline $\mathrm{TC}>5.2(\mathrm{mmol} / \mathrm{l})$ & $1.24(0.79,1.96)$ & 0.36 & & \\
\hline TG > $1.7(\mathrm{mmol} / \mathrm{l})$ & $1.18(0.86,1.63)$ & 0.31 & & \\
\hline $\mathrm{HDL}-\mathrm{c}<1.0(\mathrm{mmol} / \mathrm{l})$ & $1.22(0.90,1.67)$ & 0.20 & & \\
\hline LDL-c > 4.1 (mmol/l) & $1.42(0.45,4.45)$ & 0.55 & & \\
\hline Dyslipidemia & $1.28(0.95,1.73)$ & 0.11 & & \\
\hline $\mathrm{HBs} \mathrm{Ag+}$ & $1.16(0.74,1.82)$ & 0.51 & & \\
\hline $\mathrm{HCV}-\mathrm{Ab}+$ & $1.69(1.05,2.72)$ & 0.03 & NA & NA \\
\hline Route of HIV transmission & & & NA & NA \\
\hline Heterosexual & $0.99(0.55,1.80)$ & 0.98 & & \\
\hline MSM & $1.21(0.67,2.17)$ & 0.53 & & \\
\hline Blood transfusion & $4.12(2.08,8.19)$ & $<0.001$ & & \\
\hline Others & Reference & & & \\
\hline $\begin{array}{l}\text { Years since HIV diagnosis } \\
\text { (per year increase) }\end{array}$ & $1.06(0.97,1.14)$ & 0.19 & & \\
\hline $\begin{array}{l}\text { Baseline CD4+ cell count } \\
\text { (per cells } / \mathrm{mm}^{3} \text { increase) }\end{array}$ & $0.999(0.997,1.000)$ & 0.03 & $1.003(1.001,1.004)$ & $<0.01$ \\
\hline $\begin{array}{l}\text { Baseline HIV Viral load } \\
\text { (per log10 copies } / \mathrm{ml} \text { increase) }\end{array}$ & $0.97(0.74,1.27)$ & 0.82 & & \\
\hline Stavudine exposure & $1.92(1.42,2.60)$ & $<0.001$ & NA & NA \\
\hline Zidovudine exposure & $0.50(0.36,0.71)$ & $<0.001$ & $0.15(0.10,0.24)$ & $<0.001$ \\
\hline TDF exposure & $0.46(0.33,0.64)$ & $<0.001$ & $0.13(0.08,0.21)$ & $<0.001$ \\
\hline $\begin{array}{l}\text { Recent CD4+ cell count } \\
\text { (per cells } / \mathrm{mm}^{3} \text { increase) }\end{array}$ & $0.996(0.995,0.997)$ & $<0.001$ & & \\
\hline Recent CD4+/CD8+ ratio & $0.09(0.05,0.17)$ & $<0.001$ & $0.14(0.06,0.31)$ & $<0.001$ \\
\hline $\begin{array}{l}\text { Recent VL } \\
\text { (per log10 copies } / \mathrm{ml} \text { increase) }\end{array}$ & $1.87(1.65,2.12)$ & $<0.001$ & $1.28(1.08,1.51)$ & $<0.01$ \\
\hline
\end{tabular}

Abbreviations: HR Hazard ratio; Cl confidence interval; NA not available; $B M I$ body mass index; $T C$ total cholesterol; TG triglycerides; $H D L-c$ high-density lipoprotein cholesterol; $L D L-C$ low-density lipoprotein cholesterol; HBsAg+ hepatitis B surface antigen; HCV Ab hepatitis C antibody; HIV human immunodeficiency virus; MSM men who have sex with men; TDF tenofovir disoproxil fumarate; VL HIV-1 viral load

during which the two cohorts were enrolled [12, 24]. The prevalence of hypertension observed in our study was also lower than that reported by Ding et al. among Chinese PLWH (23.8\%), however that study was carried out in a single study site in Zhejiang province, and included both ART-naïve and ART-experienced PLWH [13].

By contrast, the incidence of hypertension in our cohort was slightly higher than that reported in the general Chinese population (7.6 vs. $5.2-5.3$ per 100 PYs) [24, 25 ]. In terms of comparisons with incidence data from
PLWH in other countries, an analysis of data from the Data collection on Adverse events of Anti-HIV Drugs (D:A:D) multi-cohort study from 1999 to 2003 found that the incidence of hypertension among PLWH in Europe, North America and Australia was 7.2 per 100 PYs [7]. However, in recent large studies from similar regions, the incidence of hypertension among PLWH was lower, and varied from 2.6 to 6.4 per 100 PYs [5, 9]. Data from Africa demonstrated incidence of hypertension was 11.2-12.0 per 100 PYs, which was higher than our 


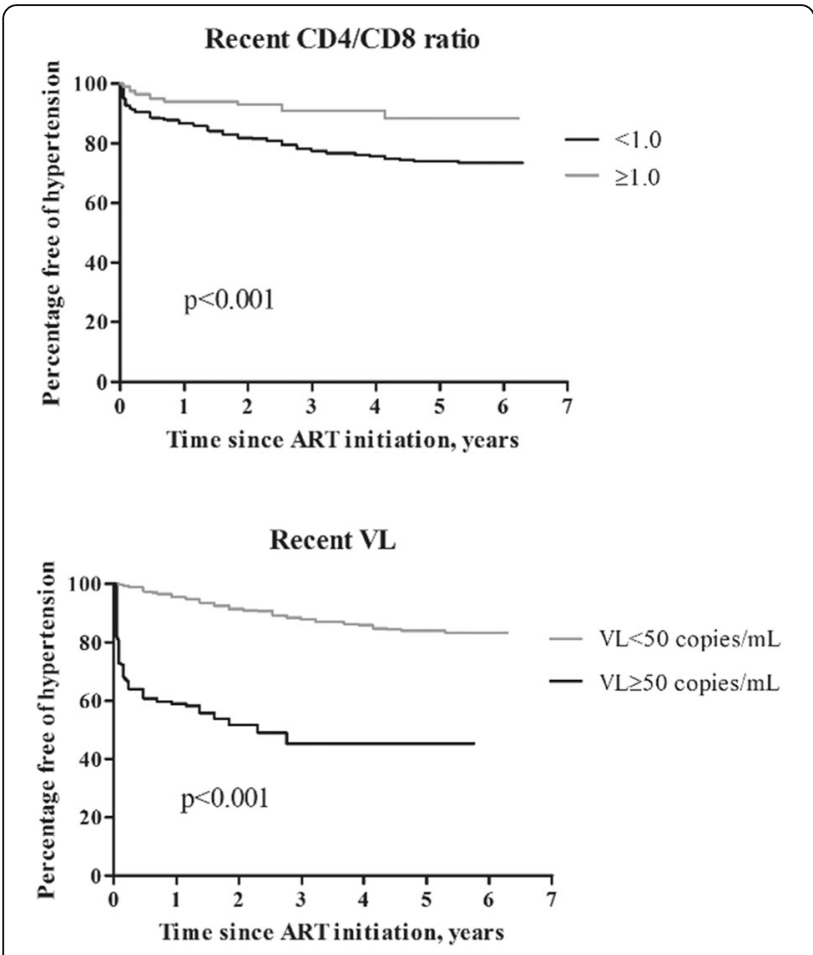

Fig. 2 Kaplan-Meier survival estimates of incident hypertension. Abbreviations: ART, antiretroviral therapy; VL, HIV-1 viral load

present study $[10,11]$, which may reflect overall higher incidence of hypertension in persons of African descent. A population-based cohort study of 6814 middle-aged and older adults from the United States demonstrated that hypertension incidence was higher among African Americans (8.5 per $100 \mathrm{PYs}$ ) compared with Caucasians (5.7 per $100 \mathrm{PYs}$ ) and Chinese Americans (5.2 per 100 PYs) [25]. Therefore, the incidence of hypertension among patients in our study was slightly higher than general Chinese population and PLWH from Europe, North America and Australia, however lower than PLWH among Africa.

Interestingly, in our study, recent $\mathrm{CD} 4+/ \mathrm{CD} 8+$ ratio among PLWH was associated with incident hypertension [Adjusted HR:0.14 (0.06, 0.31)]. CD4+/CD8+ ratio has been used in prior studies as a strong marker of immune activation and immune senescence [26], and a lower CD4+/CD8+ ratio during ART has been shown to predict residual plasma viremia of $\geq 1.0$ copy $/ \mathrm{ml}$ [27] among virologically suppressed PLWH. Further evidence has demonstrated that low $\mathrm{CD} 4+$ / CD8+ ratios among PLWH are associated with neurocognitive disorders, lung cancer, pulmonary emphysema, and AIDS-related mortality [28-31]. No prior studies have examined the relationship between hypertension and $\mathrm{CD} 4+/ \mathrm{CD} 8+$ ratios among PLWH. An abstract from the 2014 HIV Drug Therapy Glasgow Congress found that low $\mathrm{CD} 4+/ \mathrm{CD} 8+$ ratio $(<0.8)$ predicted higher cardiovascular risk [32], and inversion of the $\mathrm{CD} 4+/ \mathrm{CD} 8+$ ratio was associated with higher carotid intima-media thickness and arterial stiffness [33]. Among PLWH, chronic T-cell activation and release of cytokines promote renal sodium and water retention, vasoconstriction, and vascular remodeling, resulting in elevated blood pressure [34]. A few studies have also demonstrated a relationship between hypertension and inflammatory biomarkers (e.g. Creactive protein, D-dimer and interleukin-6), immune activation and microbial translocation [35-37]. These data, combined with our findings of an association between low recent $\mathrm{CD} 4+/ \mathrm{CD} 8+$ ratio and increased incidence of hypertension among PLWH, supports the need for additional studies aimed at confirming and better understanding the potential mechanisms behind this relationship.

A novel finding in this present study was that persistent HIV viremia was associated with incident hypertension among PLWH. Okeke et al. previously reported that HIV-related immunosuppression and ongoing viral replication may contribute to a higher risk of hypertension [8]; however in their study, no statistically significant differences were observed for the parameters of HIV viremia. Two studies from the United States and Europe also did not identify an association between HIV $\mathrm{VL}$ and the incidence of hypertension [4, 5]. However, these studies differed from ours with respect to the ethnic background of the participants and statistical approach. Prior studies have demonstrated the direct effects of HIV proteins, chronic inflammation induced by HIV-1 infection, and metabolic effects of ART contribute to endothelial dysfunction and atherosclerotic disease among PLWH $[38,39]$. Endothelial dysfunction impairs endothelium-dependent vasodilatation, and causes alterations in the interactions between endothelium and leukocytes, thrombocytes and regulatory molecules, ultimately leading to hypertension [40].

Data regarding the relationship between specific antiretrovirals and hypertension have yielded conflicting results. Some studies have found that exposure to protease inhibitors, especially lopinavir/ritonavir, is associated with development of hypertension [41, 42], which may be attributable to activation of the adipocyte reninangiotensin system [43]. Nduka et al. reported that stavudine-induced body composition change was associated with higher prevalence of hypertension [15]. Another longitudinal study demonstrated that prolonged exposure to both non-nucleoside reverse transcriptase inhibitors (NNRTI) and protease inhibitors was a risk factor of hypertension [44]. Several potential mechanisms have been hypothesized to explain the association between ART and hypertension, including gain in body weight, immune reconstitution, 
and endothelial dysfunction [41]. One animal model study showed zidovudine could increase blood pressure and promote cardiovascular damage through a $\mathrm{NAD}(\mathrm{P}) \mathrm{H}$ oxidase-dependent mechanism [45]. By contrast, other studies have found no association between exposure to protease inhibitors, stavudine and NNRTIs on the development of hypertension $[5,8,11]$, and data from the D:A:D study demonstrated cumulative exposure to NNRTIs was associated with a decreased risk of incident hypertension [7]. In the present study, stavudine was not found to be associated with incident hypertension, possibly due to the relatively short length of exposure of stavudine and fewer body composition changes [16]. Our data also demonstrated TDF or zidovudine exposure were protective factors for incident hypertension among PLWH, which is discordant with the aforementioned studies [11, 42]. However, a retrospective longitudinal study from Brazil found that cumulative exposure of TDF or zidovudine were associated with lower cardiovascular event risk [46], which was consistent with our finding. The nuances of the relationship between ART and hypertension require further research.

In our univariate analysis, baseline $\mathrm{CD} 4+$ cell count was not associated with hypertension incidence [Crude HR:0.999 $(0.997,1.000)]$, however in the adjusted model, baseline CD4+ cell count was associated with hypertension incidence [Adjusted HR:1.004 (1.002,1.006)]. Given the weak magnitude of the relationships observed in this regard, we feel this finding does not likely carry strong clinical implications for our study population and should not be over-interpreted.

Our study has a few limitations. First, our study population represents patients enrolled in two multi-center prospective studies of treatment-naïve patients initiating government-sponsored, currently available ART regimens at the time of enrollment. Therefore, our findings may be influenced by differences in the two studies. However, we did not detect a significant difference in the incidence rates between patients in the two groups, and given the patients were recruited from study sites within the same research network there was a high degree of consistency in the enrollment criteria and technique for study evaluations. We also acknowledge that the sample does not fully represent the entire population of patients with HIV in China, as participants were able to meet criteria for entry into the study and maintain follow-up care. However, if anything, we expect that this would underestimate the magnitude of our findings. Furthermore, while our sample size was smaller compared with studies from European and the United States $[5,7-9,11]$, it represents the largest study to date in Asia to explore this important issue. In addition, it is possible that a few people classified as having hypertension based on taking an antihypertension medication maybe have been misclassified, as these medications may be used to treat other conditions. Finally, the follow-up period in the present study is comparatively short, and therefore continued follow up is needed to understand how incidence rates change after longer periods of exposure to ART.

\section{Conclusions}

Hypertension is a major modifiable risk factor for cardiovascular disease, an important cause of mortality among PLWH. We found that incidence of hypertension was high compared with rates among the general population. Recent low $\mathrm{CD} 4+/ \mathrm{CD} 8+$ ratio and detectable HIV viremia were associated with incident hypertension, while receipt of ART was associated with reduced risk. Hypertension may be mitigated, in part, by excellent HIV care, including viral suppression with ART.

\section{Abbreviations}

aHR: Adjusted hazard ratio; ART: Antiretroviral therapy; AZT: Zidovudine; BMI: Body mass index; CACT: China AIDS Clinical Trial; Cl: Confidence interval; d4T: Stavudine; EFV: Efavirenz; eGFR: Estimated glomerular filtration rate; HBsAg: Hepatitis B virus surface antigen; HBV: Hepatitis B virus; HCV: Hepatitis C virus; HCV Ab: Hepatitis C virus antibody; HDL-C: High-density lipoprotein cholesterol; HR: Hazard ratios; MSM: Men who have sex with men; NNRTI: Non-nucleoside reverse transcriptase inhibitors; NVP: Nevirapine PLWH: Persons living with HIV; PUMCH: Peking Union Medical College Hospital; PYs: Person-years; 3TC: Lamivudine; TDF: Tenofovir disoproxil fumarate; VL: Viral load

\section{Acknowledgements}

We thank the patients and their families for their participation and support during this study. The following clinical institutions or hospitals participated in this study: The Infectious Disease Hospital of Henan Province (Qingxia Zhao); Shanghai Public Health Clinical Center Affiliated to Fudan University (Li Liu); Fuzhou Infectious Diseases Hospital Affiliated to Fujian Medical University (Hanhui Ye); Guangzhou No. 8 People's Hospital (Xiaoping Tang); Shenzhen Third People's Hospital (Hui Wang, Yun He); Beijing You'an Hospital, Capital Medical University (Hao Wu); Beijing Ditan Hospital, Capital Medical University (Hongxin Zhao); Yunnan AIDS Care Center (Huiqin Li); The First People's Hospital of Honghe State (Song Huo); Tangdu Hospital Affiliated to the Fourth Military Medical University, Xi'an (Yongtao Sun); The Fourth People's Hospital of Nanning, Guangxi (Shaobiao Huang); 302 Military Hospital, Beijing (Weiwei Chen); Guangxi Center for Disease Prevention and Control, Nanning, Guangxi (Yongzhen Li); Chengdu Infectious Diseases Hospital (Shenghua He); Longtan Hospital of Guangxi Zhuang Autonomous Region (Zhihao Meng); The First Hospital of Changsha (Min Wang); The First Affiliated Hospital of China Medical University (Jing Liu). We would like to recognize the following members of the $\mathrm{PUMCH}$ research team for their overall contributions to the cohort study: Zhifeng Qiu, Zhenyin Liu, and Wei Cao. Finally, special thanks to the Yale Center for Interdisciplinary Research on AIDS, Sten H. Vermund (Yale School of Public Health, New Haven, CT, USA), and J. Lucian Davis (Yale School of Public Health, New Haven, CT, USA) for their valuable input during the preparation of this manuscript.

\section{Authors' contributions}

FHW, GFP, and LTS participated in the conception and design of the study. FHW and GFP performed the data analysis and drafted the manuscript. HE and CWT contributed to the analytic approach, writing, and revising of the manuscript. LW, HY, XJ, LYL, and SXJ performed the original data collection (including patient recruitment, laboratory analyses and clinical data collection) and provided direct input to development of the methodology section for this manuscript. LTS supervised this study. All authors reviewed and approved the final version of the manuscript. 


\section{Funding}

This study was funded by the National Key Technologies Research \& Development Program for the 11th Five-year Plan (2008ZX1001-006), the 12th Five-year Plan (2012ZX10001003-001) and the 13th Five-year Plan (2017ZX10202101). This study was also funded by Chinese Academy of Medical Sciences (CAMS) Innovation Fund for Medical Sciences (CAMS-12 M) (2017-12 M-1-014). Dr. Hsieh is supported by NIH/Fogarty International Center K01TW009995, and the Yale Center for Clinical Investigation/Doris Duke Foundation Fund to Retain Clinical Scientists. The funding bodies had no involvement in the design of the study, collection, analysis or interpretation of data, or in writing the manuscript.

\section{Availability of data and materials}

The datasets used and/or analyzed during the current study are available from the corresponding author on reasonable request.

\section{Ethics approval and consent to participate}

Peking Union Medical College Hospital (PUMCH) in Beijing, China served as the primary study center for both studies, and training and monitoring of study sites was overseen by the same contract research organization. Both protocols were approved by the PUMCH institutional review board prior to initiation of study activities. All study participants provided written informed consent at the time of enrollment, and all procedures were performed in compliance with the ethical standards of The Declaration of Helsinki.

\section{Consent for publication}

Not applicable.

\section{Competing interests}

Dr. Hsieh has received honoraria from Gilead. The remaining authors declare that they have no competing interests.

\section{Author details}

${ }^{1}$ Department of Infectious Diseases, Peking Union Medical College Hospital, Chinese Academy of Medical Sciences, Beijing, China. ${ }^{2}$ Department of Internal Medicine, Yale School of Medicine, New Haven, CT, USA. ${ }^{3}$ School of Nursing, University of California, Los Angeles, CA, USA. ${ }^{4}$ Center for AIDS Research, Chinese Academy of Medical Sciences and Peking Union Medical College, Beijing, China

\section{Received: 20 December 2019 Accepted: 25 March 2020}

Published online: 01 June 2020

\section{References}

1. Smit M, Brinkman K, Geerlings S, Smit C, Thyagarajan K, Sighem A, et al. Future challenges for clinical care of an ageing population infected with HIV: a modelling study. Lancet Infect Dis. 2015;15(7):810-8.

2. Armah KA, Chang CC, Baker JV, Ramachandran VS, Budoff MJ, Crane HM, et al. Prehypertension, hypertension, and the risk of acute myocardial infarction in HIV-infected and -uninfected veterans. Clin Infect Dis. 2014; 58(1):121-9

3. Xu Y, Chen X, Wang K. Global prevalence of hypertension among people living with HIV: a systematic review and meta-analysis. J Am Soc Hypertens. 2017;11(8):530-40.

4. Manner IW, Baekken M, Oektedalen O, Os I. Hypertension and antihypertensive treatment in HIV-infected individuals. A longitudinal cohort study. Blood Press. 2012;21(5):311-9.

5. Krauskopf K, Van Natta ML, Danis RP, Gangaputra S, Ackatz L, Addessi A, et al. Correlates of hypertension in patients with AIDS in the era of highly active antiretroviral therapy. J Int Assoc Provid AIDS Care. 2013;12(5):325-33.

6. De Socio GV, Ricci E, Maggi P, Parruti G, Celesia BM, Orofino G, et al. Time trend in hypertension prevalence, awareness, treatment, and control in a contemporary cohort of HIV-infected patients: the HIV and hypertension study. J Hypertens. 2017;35(2):409-16.

7. Thiebaut R, El-Sadr WM, Friis-Moller N, Rickenbach M, Reiss P, Monforte AD, et al. Predictors of hypertension and changes of blood pressure in HIVinfected patients. Antivir Ther. 2005;10(7):811-23.

8. Okeke NL, Davy T, Eron JJ, Napravnik S. Hypertension among HIV-infected patients in clinical care, 1996-2013. Clin Infect Dis. 2016;63(2):242-8.

9. Wong C, Gange SJ, Buchacz K, Moore RD, Justice AC, Horberg MA, et al. First occurrence of diabetes, chronic kidney disease, and hypertension among north American HIV-infected adults, 2000-2013. Clin Infect Dis. 2017; 64(4):459-67.

10. Rodriguez-Arboli E, Mwamelo K, Kalinjuma AV, Furrer H, Hatz C, Tanner M, et al. Incidence and risk factors for hypertension among HIV patients in rural Tanzania - a prospective cohort study. PLoS One. 2017;12(3):e0172089.

11. Okello S, Kanyesigye M, Muyindike WR, Annex BH, Hunt PW, Haneuse S, et al. Incidence and predictors of hypertension in adults with HIV-initiating antiretroviral therapy in South-Western Uganda. J Hypertens. 2015;33(10): 2039-45.

12. Guo F, Hsieh E, Lv W, Han Y, Xie J, Li Y, et al. Cardiovascular disease risk among Chinese antiretroviral-naive adults with advanced HIV disease. BMC Infect Dis. 2017;17(1):287.

13. Ding $Y$, Lin H, Liu $X$, Zhang $Y$, Wong FY, Sun $Y$, et al. Hypertension in HIVinfected adults compared with similar but uninfected adults in China: body mass index-dependent effects of nadir CD4 count. AIDS Res Hum Retrovir. 2017;33(11):1117-25

14. Hejazi N, Huang MS, Lin KG, Choong LC. Hypertension among HIV-infected adults receiving highly active antiretroviral therapy (HAART) in Malaysia. Glob J Health Sci. 2013:6(2):58-71.

15. Nduka CU, Stranges S, Sarki AM, Kimani PK, Uthman OA. Evidence of increased blood pressure and hypertension risk among people living with HIV on antiretroviral therapy: a systematic review with meta-analysis. J Hum Hypertens. 2016;30(6):355-62.

16. Li T, Guo F, Li Y, Zhang C, Han Y, Lye W, et al. An antiretroviral regimen containing 6 months of stavudine followed by long-term zidovudine for first-line HIV therapy is optimal in resource-limited settings: a prospective, multicenter study in China. Chin Med J. 2014;127(1):59-65.

17. Pickering TG, Hall JE, Appel LJ, Falkner BE, Graves J, Hill MN, et al. Recommendations for blood pressure measurement in humans and experimental animals: part 1: blood pressure measurement in humans: a statement for professionals from the Subcommittee of Professional and Public Education of the American Heart Association Council on high blood pressure research. Hypertension. 2005;45(1):142-61.

18. Clinical Guidelines on the Identification, Evaluation, and Treatment of Overweight and Obesity in Adults--The Evidence Report. National Institutes of Health. Obesity research. 1998:6 Suppl 2:51S-2095.

19. National Cholesterol Education Program Expert Panel on Detection E, Treatment of High Blood Cholesterol in A. Third Report of the National Cholesterol Education Program (NCEP) Expert Panel on Detection, Evaluation, and Treatment of High Blood Cholesterol in Adults (Adult Treatment Panel III) final report. Circulation. 2002;106(25):3143-421.

20. Levey AS, Stevens LA, Schmid CH, Zhang YL, Castro AF 3rd, Feldman HI, et al. A new equation to estimate glomerular filtration rate. Ann Intern Med. 2009;150(9):604-12.

21. Inker $L A$, Astor $B C$, Fox $C H$, Isakova $T$, Lash JP, Peralta CA, et al. KDOQI US commentary on the $2012 \mathrm{KDIGO}$ clinical practice guideline for the evaluation and management of CKD. Am J Kidney Dis. 2014;63(5):713-35.

22. van Zoest RA, van den Born BH, Reiss P. Hypertension in people living with HIV. Curr Opin HIV AIDS. 2017:12(6):513-22.

23. Yang ZJ, Liu J, Ge JP, Chen L, Zhao ZG, Yang WY, et al. Prevalence of cardiovascular disease risk factor in the Chinese population: the 2007-2008 China National Diabetes and metabolic disorders study. Eur Heart J. 2012; 33(2):213-20.

24. Liang Y, Liu R, Du S, Qiu C. Trends in incidence of hypertension in Chinese adults, 1991-2009: the China health and nutrition survey. Int J Cardiol. 2014; 175(1):96-101.

25. Carson AP, Howard G, Burke GL, Shea S, Levitan EB, Muntner P. Ethnic differences in hypertension incidence among middle-aged and older adults: the multi-ethnic study of atherosclerosis. Hypertension. 2011;57(6):1101-7.

26. Bruno G, Saracino A, Monno L, Angarano G. The revival of an "old" marker: CD4/CD8 ratio. AIDS Rev. 2017;19(2):81-8.

27. Riddler SA, Aga E, Bosch RJ, Bastow B, Bedison M, Vagratian D, et al. Continued slow decay of the residual plasma Viremia level in HIV-1-infected adults receiving long-term antiretroviral therapy. J Infect Dis. 2016;213(4):556-60.

28. Vassallo M, Durant J, Lebrun-Frenay C, Fabre R, Ticchioni M, Andersen S, et al. Virologically suppressed patients with asymptomatic and symptomatic HIV-associated neurocognitive disorders do not display the same pattern of immune activation. HIV Med. 2015:16(7):431-40.

29. Sigel K, Wisnivesky J, Crothers K, Gordon K, Brown ST, Rimland D, et al. Immunological and infectious risk factors for lung cancer in US veterans with HIV: a longitudinal cohort study. Lancet HIV. 2017;4(2):e67-73. 
30. Triplette M, Attia EF, Akgun KM, Soo Hoo GW, Freiberg MS, Butt AA, et al. A low peripheral blood $C D 4 / C D 8$ ratio is associated with pulmonary emphysema in HIV. PLoS One. 2017;12(1):e0170857.

31. Trickey A, May MT, Schommers P, Tate J, Ingle SM, Guest JL, et al. CD4:CD8 ratio and $\mathrm{CD} 8$ count as prognostic markers for mortality in human immunodeficiency virus-infected patients on antiretroviral therapy: the antiretroviral therapy cohort collaboration (ART-CC). Clin Infect Dis. 2017; 65(6):959-66.

32. Menozzi M, Zona S, Santoro A, Carli F, Stentarelli C, Mussini C, et al. CD4/ CD8 ratio is not predictive of multi-morbidity prevalence in HIV-infected patients but identify patients with higher CVD risk. J Int AIDS Soc. 2014;17(4 Suppl 3):19709.

33. Serrano-Villar S, Moreno S, Fuentes-Ferrer M, Sanchez-Marcos C, Avila M, Sainz T, et al. The CD4:CD8 ratio is associated with markers of ageassociated disease in virally suppressed HIV-infected patients with immunological recovery. HIV Med. 2014;15(1):40-9.

34. Harrison DG, Guzik TJ, Lob HE, Madhur MS, Marvar PJ, Thabet SR, et al. Inflammation, immunity, and hypertension. Hypertension. 2011;57(2):132-40.

35. van Zoest RA, Wit FW, Kooij KW, van der Valk M, Schouten J, Kootstra NA, et al. Higher prevalence of hypertension in HIV-1-infected patients on combination antiretroviral therapy is associated with changes in body composition and prior Stavudine exposure. Clin Infect Dis. 2016;63(2):205-13.

36. Okello S, Asiimwe SB, Kanyesigye M, Muyindike WR, Boum Y 2nd, Mwebesa $\mathrm{BB}$, et al. D-dimer levels and traditional risk factors are associated with incident hypertension among HIV-infected individuals initiating antiretroviral therapy in Uganda. J Acquir Immune Defic Syndr. 2016;73(4):396-402.

37. Manner IW, Baekken M, Kvale D, Oektedalen O, Pedersen M, Nielsen SD, et al. Markers of microbial translocation predict hypertension in HIV-infected individuals. HIV Med. 2013;14(6):354-61.

38. Mazzuca P, Caruso A, Caccuri F. HIV-1 infection, microenvironment and endothelial cell dysfunction. New Microbiol. 2016;39(3):163-73.

39. Piconi S, Parisotto S, Rizzardini G, Passerini S, Meraviglia P, Schiavini M, et al. Atherosclerosis is associated with multiple pathogenic mechanisms in HIVinfected antiretroviral-naive or treated individuals. AIDS. 2013;27(3):381-9.

40. Konukoglu D, Uzun H. Endothelial dysfunction and hypertension. Adv Exp Med Biol. 2017;956:511-40.

41. Crane HM, Van Rompaey SE, Kitahata MM. Antiretroviral medications associated with elevated blood pressure among patients receiving highly active antiretroviral therapy. AIDS. 2006;20(7):1019-26.

42. Peck RN, Shedafa R, Kalluvya S, Downs JA, Todd J, Suthanthiran M, et al. Hypertension, kidney disease, HIV and antiretroviral therapy among Tanzanian adults: a cross-sectional study. BMC Med. 2014;12:125.

43. Boccara F, Auclair M, Cohen A, Lefevre C, Prot M, Bastard JP, et al. HIV protease inhibitors activate the adipocyte renin angiotensin system. Antivir Ther. 2010;15(3):363-75.

44. Tripathi A, Jerrell JM, Skelton TN, Nickels MA, Duffus WA. Incidence of primary hypertension in a population-based cohort of HIV-infected compared with non-HIV-infected persons and the effect of combined antiretroviral therapy. J Am Soc Hypertens. 2015;9(5):351-7.

45. Papparella I, Ceolotto G, Berto L, Cavalli M, Bova S, Cargnelli G, et al. Vitamin $C$ prevents zidovudine-induced $\mathrm{NAD}(\mathrm{P}) \mathrm{H}$ oxidase activation and hypertension in the rat. Cardiovasc Res. 2007;73(2):432-8.

46. Diaz CM, Segura ER, Luz PM, Clark JL, Ribeiro SR, De Boni R, et al. Traditional and HIV-specific risk factors for cardiovascular morbidity and mortality among HIV-infected adults in Brazil: a retrospective cohort study. BMC Infect Dis. 2016;16:376

\section{Publisher's Note}

Springer Nature remains neutral with regard to jurisdictional claims in published maps and institutional affiliations.

Ready to submit your research? Choose BMC and benefit from:

- fast, convenient online submission

- thorough peer review by experienced researchers in your field

- rapid publication on acceptance

- support for research data, including large and complex data types

- gold Open Access which fosters wider collaboration and increased citations

- maximum visibility for your research: over $100 \mathrm{M}$ website views per year

At $\mathrm{BMC}$, research is always in progress.

Learn more biomedcentral.com/submissions 